\title{
Ultrasound Diagnosis of Evolving Pyloric Stenosis
}

\author{
Deborah A. Weiskittel, Dana L. Leary, and Caroline E. Blane \\ Department of Radiology, Section of Pediatric Radiology, University of Michigan Medical Center, Ann Arbor, \\ Michigan, USA
}

\begin{abstract}
Two infants with recurrent episodes of vomiting had upper gastrointestinal barium studies that did not show radiographic features of pyloric stenosis. However, follow-up abdominal sonograms done 1-2 weeks later documented hypertrophic pyloric stenosis, which led to surgery. This entity is not congenital, but an evolving acquired lesion. Therefore, ultrasound is an excellent modality to evaluate and monitor patients clinically suspected of developing hypertrophic pyloric stenosis despite their initially negative imaging studies.
\end{abstract}

Key words: Abdomen, sonography - Hypertrophic pyloric stenosis, diagnosis.

Hypertrophic pyloric stenosis is a common problem in infancy, affecting $1: 150$ boys and $1: 775$ girls [1]. The patients typically have a normal postnatal course, and then present with nonbilious projectile vomiting at several weeks of age [2]. Clinical diagnosis through the palpation of the hypertrophic muscle layers, the "olive," can be difficult, and often the diagnosis rests on radiographic imaging. There have been several patients whose initial radiographic examination was negative or equivocal for hypertrophic pyloric stenosis, but later were found to have the disease. Two such cases are herein described.

\section{Case Reports}

Case 1

A 6-week-old boy with a 4-week history of projectile vomiting had an upper gastrointestinal barium study that showed some

Address reprint requests to: Caroline E. Blane, M.D., Pediatric Radiology, Mott Children's Hospital (C3123/0252), 1500 E. Medical Center Drive, Ann Arbor, MI 48109, USA pylorospasm and gastroesophageal reflux (Fig. 1). He was started on thickened formula and improved slightly, but projectile vomiting soon recurred. Two weeks later with a continued history of vomiting, the clinical suspicion of pyloric stenosis was again entertained, although no pyloric mass could be palpated. An abdominal ultrasound was recommended, which showed hypertrophic pyloric stenosis (Fig. 2A, B). On surgical consultation after the ultrasound, a mass was thought to be palpated in the right upper quadrant. He underwent pyloromyotomy the next day and was discharged on the first postoperative day without complications.

\section{Case 2}

A 34-week-gestation boy had spent the first 3 months of his life in a neonatal intensive care unit due to various congenital problems. Feeding was initiated through an orogastric tube. At 6 weeks of age he required suctioning every $1-2 \mathrm{~h}$ for retained gastric contents. An upper gastrointestinal series was performed at $7^{1} / 2$ weeks of age to evaluate for gastroesophageal reflux and gastric emptying. Pylorospasm and elongation of

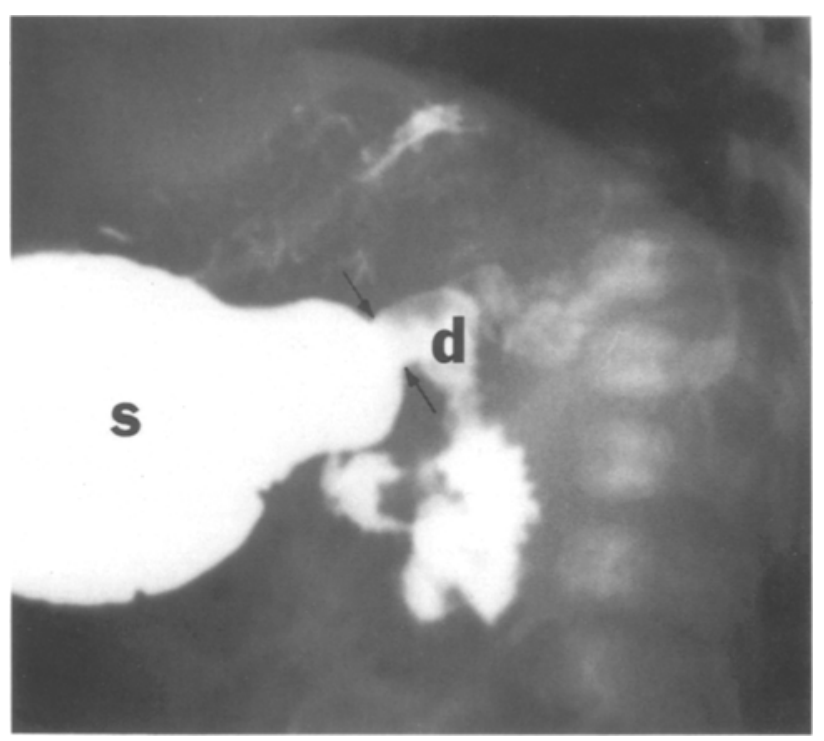

Fig. 1. Lateral view of the upper gastrointestinal tract in this 6-week-old infant shows the normal pylorus (arrows), stomach $(s)$, and duodenal bulb $(d)$. 

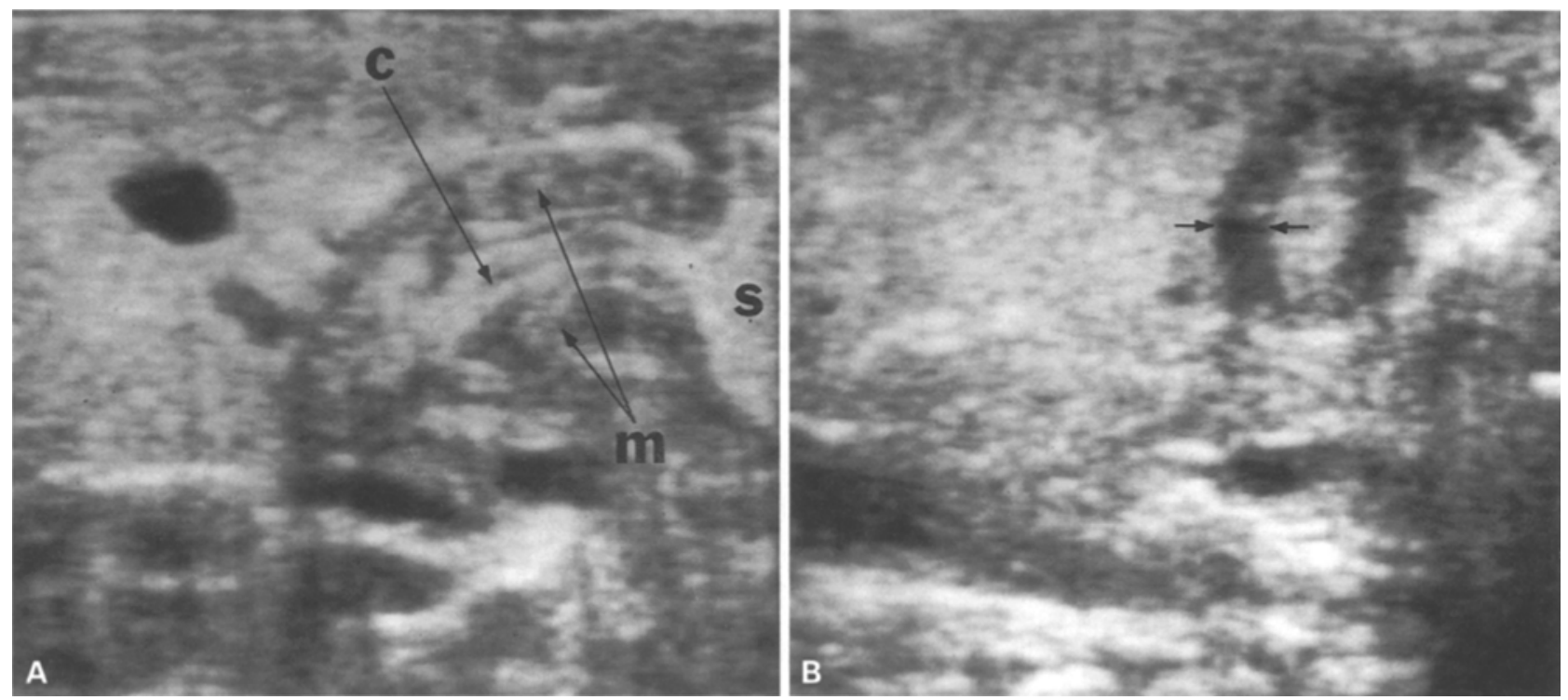

Fig. 2. A Longitudinal sonogram of the pylorus 14 days later. The canal $(c)$ is elongated and surrounded by the hypertrophied muscle $(m)$ distal to the gastric antrum $(s)$. B Transverse sonogram showing the hypertrophied pyloric muscle (arrows).

the pyloric channel were noted, but no definite muscle mass of pyloric stenosis was identified. Gastric atony with retained secretions was present. The child continued having large amounts of gastric residuals following feeding. Six days later an abdominal ultrasound was performed, which showed hypertrophic pyloric stenosis. An uncomplicated pyloromyotomy was performed 3 days later.

\section{Discussion}

While the etiology of pyloric stenosis remains obscure, it is known that hypertrophic pyloric stenosis is not present at birth [1]. The average age of onset of hypertrophic pyloric stenosis is between 2 and 6 weeks, peaking in the third week after birth [2]. Ultrasound has been used to image the hypertrophied muscle [3], and recent studies have discussed the upper limits of normal for pyloric muscle and length measurements [4]. More recently, Tunell and Wilson attempted to determine the accuracy of the pyloric muscle length in the diagnosis of hypertrophic pyloric stenosis by ultrasound [5]. By using the criteria of $1.8 \mathrm{~cm}$ or less as normal for pyloric muscle length, they had 2 false negative examinations and no false positive examinations [5]. The authors noted that these 2 patients were found to have a positive examination on a second ultrasound study 4 and 5 days after the initial examination. By denoting the initial sonograms of these 2 patients as false negatives, Tunell and Wilson are implying that these studies should have been positive because the patients later were diagnosed as having hypertrophic pyloric stenosis. An alternate interpretation of these results is that these patients had pyloric stenosis in evolution. At the time of their initial examination, their pyloric muscles had not hypertrophied enough to be outside the normal variations in length. Thus, it is not that the criteria used for distinguishing between a normal and a hypertrophied muscle are inaccurate, but that at an early point in the disease process, minimal hypertrophy may mimic the upper ranges of normal. In fact, some authors recommend assessing the overall appearance of the pylorus rather than relying upon absolute measurements [6].

If a child comes to medical attention soon after vomiting begins it may be too early to detect hypertrophic pyloric stenosis by physical examination or imaging study [7]. In our 2 cases, the initial barium study of the gastrointestinal tract was either negative for pyloric stenosis or equivocal. Neither patient had a clinically palpable muscle mass in the region of the pylorus at presentation. Case 1 had evidence of pylorospasm and gastroesophageal reflux, both possible causes for his vomiting. In both patients the follow-up ultrasound, performed between 6-14 days later, unequivocally revealed hypertrophic pyloric stenosis. It is important to remember that pyloric stenosis is an evolving disease, making it difficult to completely exclude hypertrophic pyloric stenosis on the basis of one negative examination when the clinical symptoms persist. Although our referring physicians have in the past regarded the sonographic diagnosis of hypertrophic pyloric stenosis with skepticism, these cases have prompted them to re- 
evaluate the modality. In addition, when pyloric stenosis is the major clinical diagnosis, ultrasound is now often requested as the initial imaging procedure. Ultrasound is an excellent modality to follow patients where the index of suspicion for pyloric stenosis remains high, as it is a simple noninvasive test without potential risks of ionizing radiation.

\section{References}

1. Wallgren A: Preclinical stage of infantile hypertrophic pyloric stenosis. Am J Dis Child 72:371-376, 1946

2. Kirks DR: Practical Pediatric Imaging, Diagnostic Radiology of Infants and Children. Boston: Little, Brown and Co, 1984, pp 643-648
3. Teele RL, Smith EH: Ultrasound in the diagnosis of idiopathic hypertrophic pyloric stenosis. N Engl J Med 296:1149, 1977

4. Stunden RJ, LeQuesne GW, Little KET: The improved ultrasound diagnosis of hypertrophic pyloric stenosis. Pediatr Radiol 16:200-205, 1986

5. Tunell WP, Wilson DA: Pyloric stenosis: diagnosis by realtime sonography, the pyloric muscle length method. $J$ Pediatr Surg 19:795-799, 1984

6. Blumhagen JD: The role of ultrasonography in the evaluation of vomiting in infants. Pediatr Radiol 16:267-270, 1986

7. Leahy PF, Farrell R, O'Donnell B: 300 infants with hypertrophic pyloric stenosis: presentation and outcome. Ir $\mathrm{Med}$ J 79:114-116, 1986

Received: March 16, 1988; accepted: April 18, 1988 